\title{
Nonlinear MIMO Impulse Responses Determination Using Pseudo Random Sequences, and Nonlinear Compensation
}

\author{
Joris Van Kerrebrouck, Haolin Li, Jochem Verbist, Guy Torfs, Johan Bauwelinck and Piet Demeester \\ Department of Information Technology (INTEC) - IDLab \\ University of Ghent - imec \\ Technologiepark 15, B-9052 Zwijnaarde, Belgium \\ Email: joris.vankerrebrouck@ugent.be
}

\begin{abstract}
This paper shows how to estimate an extended polynomial matrix description of a non-linear MIMO system. Both linear and non-linear impulse responses are obtained by transmitting pseudo random binary sequence (PRBS) testpatterns and can be easily separated thanks to the shift-and-add property of PRBS. Further on, a method to equalize such nonlinear systems based on this extended polynomial matrix and the result is compared with a linear zero-forcing equalizer for different channel impairments and in a VPI simulation. This proves the presented approach is suited to cancel self-phase modulation in coherent optical communication systems.
\end{abstract}

\section{INTRODUCTION}

Communication channels are pushed to maximize the data throughput, which is usually limited by the finite frequency spectrum of these mediums. To overcome this, new communication systems tend towards modulation schemes with higher spectral efficiency and/or stretch the channel bandwidth with equalizers. Therefore, channel estimation and compensation are of crucial importance. Not only are modern communication channels browbeaten to transfer more data, multiple channels are created to establish multiple data streams. Polarization division multiplexing (PDM) and space-division multiplexing (SDM) technologies are used in multiple-input multiple-output (MIMO) systems to achieve multiple parallel data streams to sustain the capacity growth [1]. Along with linear intersymbol interference (ISI) and co-channel interference (CCI), nonlinear mixing effects are also present, making the impulse responses of MIMO systems with nonlinear effects complicated. For example, the capacity of optical MIMO channels is rapidly approaching the capacity limit imposed by these nonlinear fiber effects [2] [3].

Channel estimation of MIMO systems is already widely used and numerous algorithms are available [4], [5]. Typically, the estimation is described using polynomial matrices. To improve a MIMO system, the orthogonal eigenvectors of the polynomial matrix can be used to achieve multiple virtual independent channels with no crosstalk. To do so, polynomial matrix singular value decomposition (PMSVD) can be used to decompose a MIMO channel matrix, which can then be used to recover the transmitted signals corrupted by the channel interference $(\mathrm{CI})$ at the receiver [6]. However, PMSVD is computationally heavy and will only result in a linear compensation.

To compensate nonlinear effects, nonlinear impulse responses, described with Volterra or power series should be canceled. However, identification of the coefficients is computationally burdensome.

In this paper, Pseudo Random Bit Sequence (PRBS), also called binary maximum length sequences (MLS) have been used as excitation to estimate, by cross correlation, the linear impulse responses of a MIMO channel. These impulse responses are used to build the polynomial matrix. However, the cross correlation of the PRBS stream also reveals additional impulse responses that can be attributed to nonlinear channel effects and that can be used to extend the polynomial matrix. This results in a more accurate channel model. Using this extended polynomial matrix, a heuristic method is presented to equalize a nonlinear MIMO system.

The structure of this paper is as follows: section II introduces an extended version of a polynomial matrix which includes extra elements relating to the non-linear impulse response. Furthermore, it is shown how all the elements of this matrix can be estimated based on the transmission of pseudo random bit sequences. Two important properties of these sequences allow to extract the different contributions from the received signals. In section III, a method to equalize a nonlinear MIMO system based on the extended polynomial matrix is proposed and to verify this method, different non-linear communication links are compensated in sections IV and $\mathrm{V}$ using the proposed non-linear equalizer. Finally, conclusions are drawn in section VI.

\section{POLYNOMIAL MATRIX REPRESENATION OF A MIMO CHANNEL}

Given a MIMO link with $n_{T}$ (number of transmitters) inputs and $n_{R}$ (number of receivers) outputs, a $n_{T} \times n_{R}$ polynomial matrix $\underline{\mathbf{C}}(Z)$ can be used to describe the channel. Fig. 1 shows an example of a $2 \times 2$ MIMO system. Each element of the matrix $\underline{\mathbf{C}}(Z)$ is a polynomial in the Z-domain. In this paper the Z-polynomials are translated into vector quantities to simplify the mathematical processing. The polynomial matrix can easily be extended to take non-linear terms into account. For example, Eq. (1) shows a polynomial matrix of a $2 \times 2$ MIMO system but extended with the product of the two inputs.

$$
\left[\begin{array}{l}
y_{1} \\
y_{2}
\end{array}\right]=\left[\begin{array}{lll}
C_{11} & C_{12} & C_{13} \\
C_{21} & C_{22} & C_{23}
\end{array}\right] *\left[\begin{array}{c}
x_{1} \\
x_{2} \\
x_{1} x_{2}
\end{array}\right]
$$

The mathematical operator $*$ represents the convolution. Eq. (1). 
MIMO Channel C

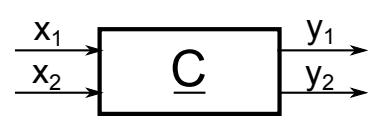

Fig. 1: Representation of a polynomial matrix in a MIMO system.

\section{A. Obtaining Impulse Response using a PRBS}

To determine the different impulse responses constituting the channel matrix $\underline{\mathrm{C}}(Z)$, a pseudo random binary sequence (PRBS) is used. A PRBS bit stream is formed using a linear feedback shift register of length $\mathrm{m}$, given a proper feedback polynomial, a maximum length sequence, with period $N=$ $2^{m}-1$ can be obtained. If the PRBS is mapped to nonreturn-to-zero (NRZ) $\{-1,+1\}$ symbols, the autocorrelation is maximal and results in $N$ when the streams are aligned and equals -1 elsewhere (see Eq. (2)). For creating a perfect Kronecker delta with an autocorrelation function, we refer to [7]. This makes it a convenient stimulus to calculate the impulse response of a channel. When a PRBS $(u(n))$ is sent through a channel and the cross correlation of the received PRBS $\left(u_{c h}(n)\right)$ with an undistorted PRBS reveals the channel impulse response $h_{c h}(n)$. This is summarized in Eq. (3)-(5)

$$
\begin{gathered}
R_{u u}(l)=\sum_{n=1}^{N} u(n) u(n-l)= \begin{cases}N & l=0 \\
-1 & l \neq 0\end{cases} \\
u_{c h}(n)=\sum_{l=-\infty}^{\infty} h_{c h}(l) u(n-l) \\
h_{c h}(l)=\sum_{n=1}^{N} u_{c h}(n) u(n-l) \\
=u_{c h}(n) * u(-n)
\end{gathered}
$$

\section{B. PRBS' Shift and Add Property}

A PRBS sequence has the property that the modulo-2 summation or 'xor' operation (denoted with $\oplus$ ) of a PRBS with a time-shifted copy of itself yields again the same PRBS but with a different time-shift. This is commonly known as the shift-and-add property [8] and is mathematically expressed in Eq. (6). If non-return-to-zero (NRZ) $\{-1,+1\}$ symbols are used, a modulo-2 sum of two symbols equals the inverse of the product of the two symbols (see Eq. (8)). Hence, multiplying two time-shifted versions of an NRZ modulated PRBS results in the same PRBS but with yet another time-shift. The timeshift or delay of the resulting PRBS depends only on the timeshift of the copy with respect to the original PRBS. Therefore, we introduce a new function $\phi($.$) in Eq. (7) to indicate the$ delay of the resulting PRBS with respect to the original PRBS. This $\phi($.$) function can be determined using Eq. (9), where we$ use a discrete logarithm in the finite Galois field of $2^{m}$. This is the same finite field used to construct the PRBS.

$$
\begin{aligned}
u(l) & =u(n) \oplus u(n-k) \\
u(n-\phi(k)) & =u(n) \oplus u(n-k) \\
& =-u(n) u(n-k) \\
\phi(k) & =\log _{G F 2^{m}}\left(1+x^{k}\right)
\end{aligned}
$$

This property can be extended for three or even more PRBS sequences, which is summarized in Eq. (10) and Eq. (11).

$$
\begin{array}{r}
u\left(n_{1}\right) u\left(n_{1}+n_{2}\right) u\left(n_{1}+n_{2}+n_{3}\right)=u\left(n_{1}+\phi\left(n_{2}, n_{3}\right)\right) \\
\phi\left(n_{2}, n_{3}\right)=\log _{G F 2^{m}}\left(1+x^{n_{2}}+x^{n_{2}+n_{3}}\right)
\end{array}
$$

C. Estimating the non-linear polynomial channel matrix of a MIMO system

Using the autocorrelation and the sum-and-add properties of a PRBS, all the elements of the channel matrix represented in Eq. (1) can be calculated. To do so, a PRBS stream $u(n)$ and a time-shifted version $u(n-k)$ are applied at the input of the channel $\left(x_{1}\right.$ and $\left.x_{2}\right)$. Thanks to the shift-and-add property of the PRBS, the product of the two inputs yields a third PRBS $u(n-\phi(k))$. This is summarized in Eq. (12)

$$
\left[\begin{array}{l}
y_{1} \\
y_{2}
\end{array}\right]=\left[\begin{array}{lll}
h_{11} & h_{12} & h_{13} \\
h_{21} & h_{22} & h_{23}
\end{array}\right] *\left[\begin{array}{c}
u(n) \\
u(n-k) \\
u(n-\phi(k))
\end{array}\right]
$$

A cross correlation with the original PRBS signals allows to estimate the different impulse responses. For example, calculating the cross correlation between $u(n)$ and $y_{2}$ will result in:

$$
\left(y_{2} * u(-n)\right)(l)=h_{21}(l)+h_{22}(l-k)+h_{23}(l-\phi(k))
$$

Hence, this results in the sum of the three impulse responses, but each centered at a different sample moment. If the temporal resolution of the PRBS stream is sufficient, the different impulse responses can be easily extracted.

\section{EQUALIZING IMPULSE RESPONSE}

The inverse channel impulse response can be used as a zero forcing equalizer. Thus, cascading the channel impulse response with the inverse channel impulse response results into a Kronecker Delta $\delta(n)$ impulse response (expressed in Eq. (14)).

$$
\delta(n)=h_{\text {equalizer }}(n) * h_{\text {channel }}(n)
$$

This equalizing polynomial matrix can be constructed using the following method. Fig. 2a shows a nonlinear $2 \times 2$ MIMO system. From this, a simplified channel model with the extra non-linear terms is constructed (Fig. 2b), following the estimation method highlighted in section II-B. To equalize the channel, the channel matrix needs to be inverted. However, this is not possible due to our construction process, as the channel matrix is not square $(2 \times 3)$. To alleviate this, the matrix is extended and a virtual output signal equal to the best linear approximation of the product of $y_{1}$ and $y_{2}$ (denoted $\widetilde{y_{12}}$ ) is added. Fig. 2c shows this. The mathematical derivation Eq. (15) shows the terms that contribute to the product $y_{1} y_{2}$. Other terms of $y_{1} y_{2}$ which do not have a $x_{1}, x_{2}$ or $x_{12}$ are neglected. Notice that $x^{2}=1$ because $x$ is a binary NRZ value $\{-1,+1\}$.

The extended channel model is given by:

$$
\begin{aligned}
y_{1} y_{2}=\underbrace{\left(h_{11} * x_{1}+h_{12} * x_{2}+h_{13} * x_{12}\right)}_{y_{1}} \\
\times \underbrace{\left(h_{21} * x_{1}+h_{22} * x_{2}+h_{23} * x_{12}\right)}_{y_{2}}
\end{aligned}
$$




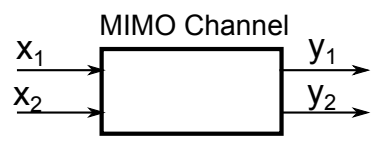

(a) 2x2 MIMO channel.

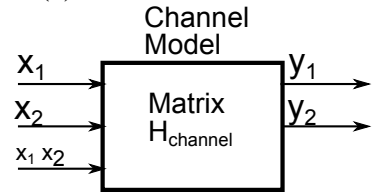

(b) Channel model with the extra non-linear term.

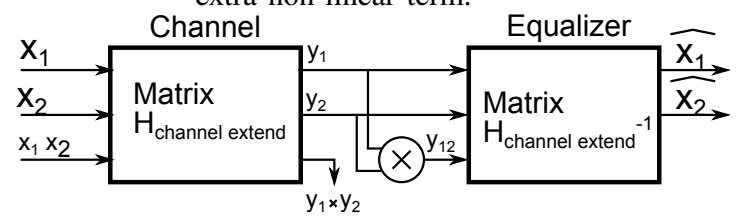

(c) $2 \times 2$ MIMO channel with equalizer.

Fig. 2: Graphical representation of the MIMO equalization algorithm.

$$
\begin{aligned}
& {\left[\begin{array}{c}
y_{1} \\
y_{2} \\
y_{12}
\end{array}\right]=} \\
& \underbrace{\left[\begin{array}{ccc}
h_{11} & h_{12} & h_{13} \\
h_{21} & h_{22} & h_{23} \\
h_{12} h_{23}+h_{13} h_{22} & h_{11} h_{23}+h_{13} h_{21} & h_{11} h_{22}+h_{12} h_{21}
\end{array}\right]}_{h_{\text {ch_extend }}} \\
& *\left[\begin{array}{c}
x_{1} \\
x_{2} \\
x_{1} x_{2}
\end{array}\right]
\end{aligned}
$$

To equalize the channel the inverse of the extended channel $h_{e q}$ is calculated. Not that $y_{12}$ represent the product of two output signals.

$$
\left[\begin{array}{l}
\hat{x_{1}} \\
\hat{x_{2}}
\end{array}\right]=h_{e q}\left[\begin{array}{c}
y_{1} \\
y_{2} \\
y_{12}
\end{array}\right]
$$

The inverted polynomial matrix is computed in the frequency domain given Eq. (17).

$$
\begin{array}{r}
{\left[\begin{array}{ccc}
\mathscr{F}\left(h_{\text {eq11 }}\right) & \mathscr{F}\left(h_{\text {eq12 }}\right) & \mathscr{F}\left(h_{\text {eq13 }}\right) \\
\mathscr{F}\left(h_{\text {eq21 }}\right) & \mathscr{F}\left(h_{\text {eq22 }}\right) & \mathscr{F}\left(h_{\text {eq } 23}\right) \\
\mathscr{F}\left(h_{\text {eq31 }}\right) & \mathscr{F}\left(h_{\text {eq32 }}\right) & \mathscr{F}\left(h_{\text {eq33 }}\right)
\end{array}\right]=} \\
\hline\left[\begin{array}{ccc}
\mathscr{F}\left(h_{\text {ideal }}\right) & 0 & 0 \\
0 & \mathscr{F}\left(h_{\text {ideal }}\right) & 0 \\
0 & 0 & \mathscr{F}\left(h_{\text {ideal }}\right)
\end{array}\right] \\
\hline\left[\begin{array}{ccc}
\mathscr{F}\left(h_{11}\right) & \mathscr{F}\left(h_{12}\right) & \mathscr{F}\left(h_{13}\right) \\
\mathscr{F}\left(h_{21}\right) & \mathscr{F}\left(h_{22}\right) & \mathscr{F}\left(h_{23}\right) \\
\mathscr{F}\left(h_{\text {ch_extend31 }}\right) & \mathscr{F}\left(h_{\text {ch_extend32 }}\right) & \mathscr{F}\left(h_{\text {ch_extend33 }}\right)
\end{array}\right]
\end{array}
$$

\section{Simulation}

The proposed equalizer is verified in Matlab. Different impaired $2 \times 2$ MIMO channels are constructed and are estimated using a PRBS9 with feedback polynomial $x^{9}+x^{5}+1$. A delay of 110 symbols is chosen between the PRBSs of the two inputs. The product of the PRBS and the delayed PRBS at the input of the system creates a third PRBS with a delay of 316 symbols relative to the first PRBS stream $\phi(110)=316$ with $\phi($.$) defined in Eq. (9). The outputs of the MIMO system$ are cross-correlated with the perfect PRBS, to attain the raw impulse response and a window function of 100 symbols long is used as mask to filter out the specific elements of the extended channel matrix shown in Eq. (16). The inverse polynomial matrix is calculated with Eq. (17) and is used to convolve with the received signal. In the simulations, two real inputs are assumed, which can easily be displayed using constellation points in a two-dimensional figure. Of course, it is clear that all proposed techniques can be easily extended towards higher dimensions.

In Table I the different simulated channel responses are shown. The first column shows the received constellation points, the second column shown the constellation points after a linear zero-forcing equalizer and the third column shows the constellation points after our proposed equalizer. It should be noted that random data transmitted to construct these constellation diagrams and not the PRBS9 sequence used to estimate the impulse responses. The simulation covers the most common MIMO channel distortions. The first distortion shown is a rotation, it can appear in single carrier modulation whereby the QPSK constellation rotates depending on the receiver local oscillator (LO) phase offset. This linear effect can be easily compensated with a rotation matrix. The second distortion comes from IQ mismatch. The third distortions are reflections that cause inter-symbol interference (ISI), this can also be compensated with a linear equalizer. A Trapezoidal constellation (Non-linear I) is only possible with a non-linear channel, the linear equalizer can not compensate the trapezoidal shape, which is visible on the constellation diagram of the signal after the linear equalizer. The fourth simulation transmits QPSK symbols with an offset $\{0,0\},\{0,1\},\{1,0\},\{1,1\}$ modulated on a single carrier. The channel is strongly saturated (Non-linear II), so that the $\{1,1\}$ symbol is compressed and moved to the origin. Only the proposed non-linear equalizer can place the 4 symbol on the correct coordinates. In the last simulation an asymmetrical PAM4 eye is shown, this effect can occur when sending PAM4 symbols through an optical link. Here we use 2 independent PRBS streams to create the PAM4 symbols, one PRBS stream represent the most significant bit (MSB) and the other the least significant bit (LSB), the aforementioned algorithm can obtain the impulse response of the MSB PRBS, the LSB PRBS, and the product of these two streams. This information can be used to uniformly spread the PAM4 symbols and optimize the 3 eyes to almost equal eye height.

\section{EXPERIMENTAL Simulation}

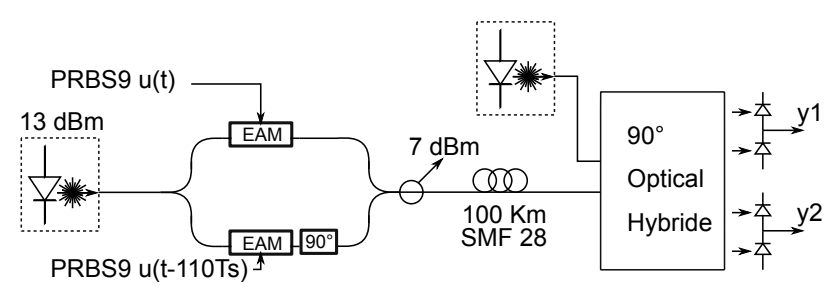

Fig. 3: VPI simulation of coherent receiver, $100 \mathrm{Km}$ fiber cause self-phase modulation (SPM). 


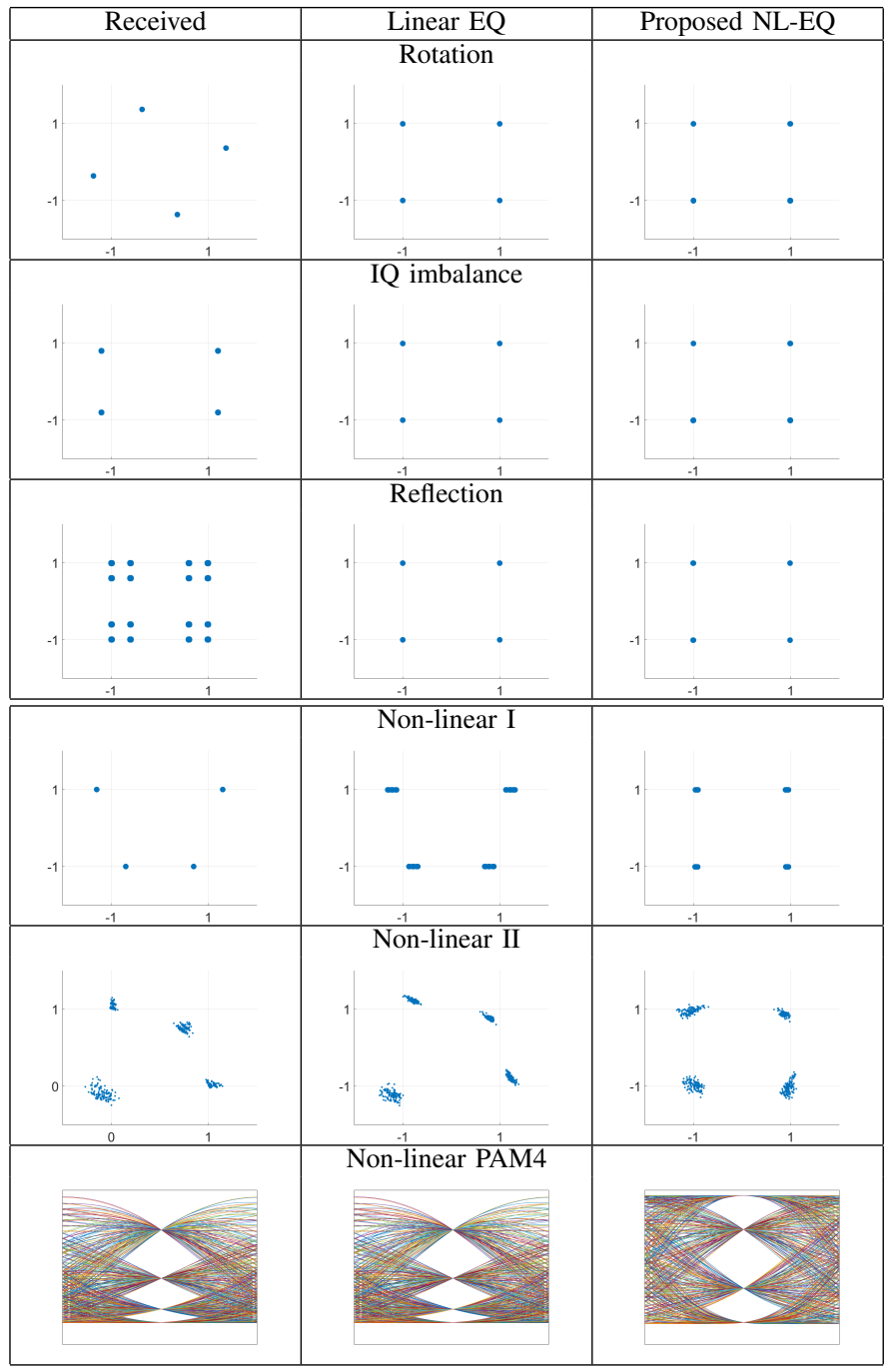

TABLE I: Matlab simulation, to verify the proposed non-linear equalizer, for the most come channel distortion effect.

The algorithm proposed in this paper can be used to describe and compensate various non-linear complex channels. To illustrate this in a more practical scenario, an optical experiment performed in VPI is set up. The simulation consists of a coherent transmitter constructed with EAMs and depicted in Fig.3. Self-phase modulation (SPM) of the signal through the fiber is one of the limited factors of the data throughput of a coherent receiver [3]. To simplify the transmitter, asymmetric QPSK symbols, namely $\{0,0\},\{0,1\},\{1,0\},\{1,1\}$ are transmitted. The QPSK symbols are made of two PRBS9 test-patterns, the second has a delay of 110 symbols. The same method is used, as described in Section IV. The PRBS test-patterns modulate with an electro-absorption modulator (EAM) the Inphase and Quadrature term of a continuous wave $(\mathrm{CW})$ laser light. The non constant envelop of the transmitted signal cause a phase fluctuation in fiber, resulting in SPM which is a form of AM-PM distortion. The fiber has a length of $100 \mathrm{Km}$ and its non-linear index $\mathrm{n} 2=2.6 \mathrm{E}-20 \frac{\mathrm{m}^{2}}{W}$, which is typical for SMF28. The light entering the fiber has a power of $7 \mathrm{dBm}$ and is modulated with a symbol rate of 1 GBaud. A scatter plot of the received QPSK constellation is shown in Table II. A linear equalizer is not capable of placing the received symbols on the right coordinates. However, the proposed non-linear equalizer can completely compensate the non-linearity.

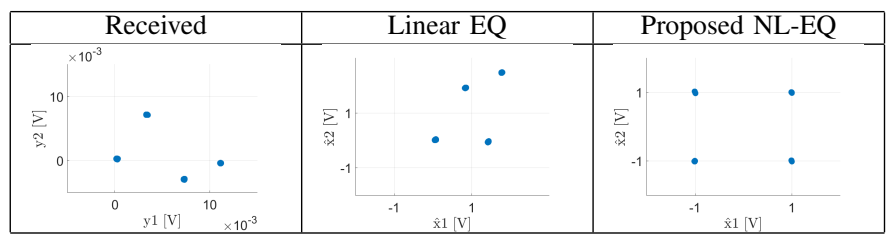

TABLE II: VPI simulation of coherent receiver, $100 \mathrm{Km}$ fiber, from left to right: the received QPSK constellation, distorted by SPM, is shown, the linear equalized received constellation and the received signal after the proposed non-linear equalizer.

\section{CONCLUSION}

This paper described an algorithm to equalize non-linear effects in a MIMO system. First, it is shown that the polynomial matrix describing a MIMO system can be extended to include non-linear effects. Second, pseudo random bit sequences are used to estimate the different elements constituting this extended polynomial matrix, and last, this estimated matrix is used to equalize data received through the non-linear channel. To show the validity of this approach, different impaired channels are simulated in Matlab and compensated with both a linear zero-forcing equalizer as well as with the proposed equalizer. It was shown that for example saturation of an unbalanced QPSK transmission could be compensated with the proposed equalizer, while this is impossible with a linear equalizer. Additional, a VPI simulation of a similar system is performed. Again an unbalanced QPSK signal is transmitted, but this time the distortion occurs due to self phase modulation. The proposed equalizer can easily compensate this. As such, it is shown that the method reported can be adjust or extend to cover multiple non-linear effects.

\section{ACKNOWLEDGMENT}

Piet Demeester thanks the ERC for his advanced grant 695495 "ATTO: A new concept for ultra-high capacity wireless networks".

\section{REFERENCES}

[1] B. Lannoo, A. Dixit, D. Colle, J. Bauwelinck, B. Dhoedt, B. Jooris I. Moerman, M. Pickavet, H. Rogier, P. Simoens, G. Torfs, D. V. Ginste, and P. Demeester, "Radio-over-fibre for ultra-small 5g cells," in 2015 17th International Conference on Transparent Optical Networks (ICTON), Jul. 2015, pp. 1-4.

[2] B. Goebel, G. J. Foschini, G. Kramer, P. J. Winzer, and R.-J. Essiambre, "Capacity Limits of Optical Fiber Networks," J. Lightw. Technol., vol. 28, no. 4, pp. 662-701, Feb. 2010.

[3] T. Mizuochi, K. Ishida, T. Kobayashi, J. Abe, K. Kinjo, K. Motoshima and K. Kasahara, "A comparative study of dpsk and ook wdm transmission over transoceanic distances and their performance degradations due to nonlinear phase noise," J. Lightw. Technol., vol. 21, no. 9, pp. 1933-1943, Sep. 2003.

[4] N. Benvenuto and G. Cherubini, Algorithms for communications systems and their applications. J. Wiley, 2002.

[5] D. Darsena, G. Gelli, and F. Verde, "Joint blind channel shortening and compensation of transmitter i/q imbalances and cfos for uplink sc-ifdma systems," Physical Communication, vol. 11, no. Supplement C, pp. $25-$ 35, 2014, radio Access Beyond $\operatorname{OFDM}(\mathrm{A})$.

[6] A. Ahrens and S. Lochmann, "Polynomial matrix algorithms for broadband optical mimo systems," in 2016 Advances in Wireless and Optical Communications (RTUWO), Nov. 2016, pp. 22-27.

[7] S. Lesecq and A. Barraud, "A prbs with exactly zero correlation and its application," in 2008 16th Mediterranean Conference on Control and Automation, Jun. 2008, pp. 724-728.

[8] Y. Lin, "'shift and add" property of m-sequences and its application to channel characterisation of digital magnetic recording," IEE Proc. Comm., vol. 142, no. 3, pp. 135-140, Jun. 1995. 